\title{
Alternative processes in the identification of familiar pictures
}

\author{
HOWARD S. HOCK, GREGORY P. GORDON, and SUSAN K. CORCORAN \\ Florida Atlantic University, Boca Raton, Florida 33431
}

\begin{abstract}
Individual differences in perceptual processing were investigated in a task requiring subjects to match words (either general or specific category names) with simultaneously presented pictures. Based on the results of previous research, individual differences in processing were inferred from the effect, on "same" reaction time, of rotating the pictures into an unfamiliar orientation. For subjects inferred to emphasize an analytic mode of processing, word-picture matches were faster for general than for specific category names, supporting the hypothesis that analytic subjects would attend to the minimal number of features necessary to infer the categorical identity of the pictures. For subjects inferred to emphasize a structural mode of processing, there was little difference in word-picture matching time between specific and general category names, supporting the hypothesis that structural subjects would attend to all the information in the pictures, regardless of the category level specified by the word.
\end{abstract}

Perception, for Bruner (1957), involves the placement of stimuli into categories on the basis of the featural information extracted from the stimuli. Our perceptual knowledge, according to Bruner, is organized such that the identity of a familiar stimulus can be inferred by attending to the minimal number of features that define the category into which the stimulus is placed. Since categories differ with respect to the number and discriminability of their defining features, it follows from Bruner's theory that different amounts of processing time may be required to place the same stimulus into different categories. Neisser (1967), though agreeing that categorization can be based on feature analysis models like Bruner's, contends that such analytic-judgmental models are too limited. He argues that "there is an unmistakeable difference between 'seeing' that two things look similar and 'judging' that they belong in the same category" (p. 95). For Neisser, what things look like depends on synthetic rather than analytic-judgmental processes.

Although Neisser (1967) never explicitly states why phenomenal experience should depend on synthetic rather than analytic processes, the point of view adopted in this study is that the completeness of phenomenal experience depends on the amount of stimulus information attended to by the perceiver. If only distinctive features are attended to, and irrelevant features ignored, in inferring the categorical identity of a stimulus, then phenomenal experience should be minimized. If, however, identification is based on the synthesis of all the detailed parts of the stimulus into a complete percept,

This research was supported by a Seed Grant awarded to the first author by Florida Atlantic University. Requests for reprints should be sent to Howard S. Hock, Department of Psychology, Florida Atlantic University, Boca Raton, Florida 33431. Gregory Gordon is currently at Emory University. and not selective attention to features that define a category, then phenomenal experience should be maximized, and processing time should not be influenced by the categorical level at which the stimulus is identified.

In the experiments reported in this study, the distinction between identification processes that minimize and maximize attention to stimulus information was examined by relating these alternative modes of stimulus identification to individual differences in mode of perceptual processing. In a series of individual difference studies involving variations on the same-different reaction time task, Hock (1973), Hock, Gordon, and Gold (1975), Hock, Gordon, and Marcus (1974), and Hock and Ross (1975) have differentiated between the structural mode of processing emphasized by some subjects and the analytic mode of processing emphasized by other subjects. Hock's distinction between structural and analytic processes is conceptually comparable with Neisser's (1967) distinction between synthetic and analytic processes. Hock's structural processes and Neisser's synthetic processes are similar in their emphasis on "putting together" or organizing the stimulus information into a complete percept (the terms "structural" and "synthetic" are hereafter used interchangeably), and both Hock and Neisser view analytic processes as decomposing the stimulus information into a set of features. It should be noted in this regard that Gestalt-type configurational properties (e.g., symmetry) can serve either a structural or an analytic function. Structurally, such properties can serve as rules for organizing the detailed parts of the stimulus into a whole. Analytically, they can function as global features for inferring the categorical identity of the stimulus.

The initial evidence for the distinction between 


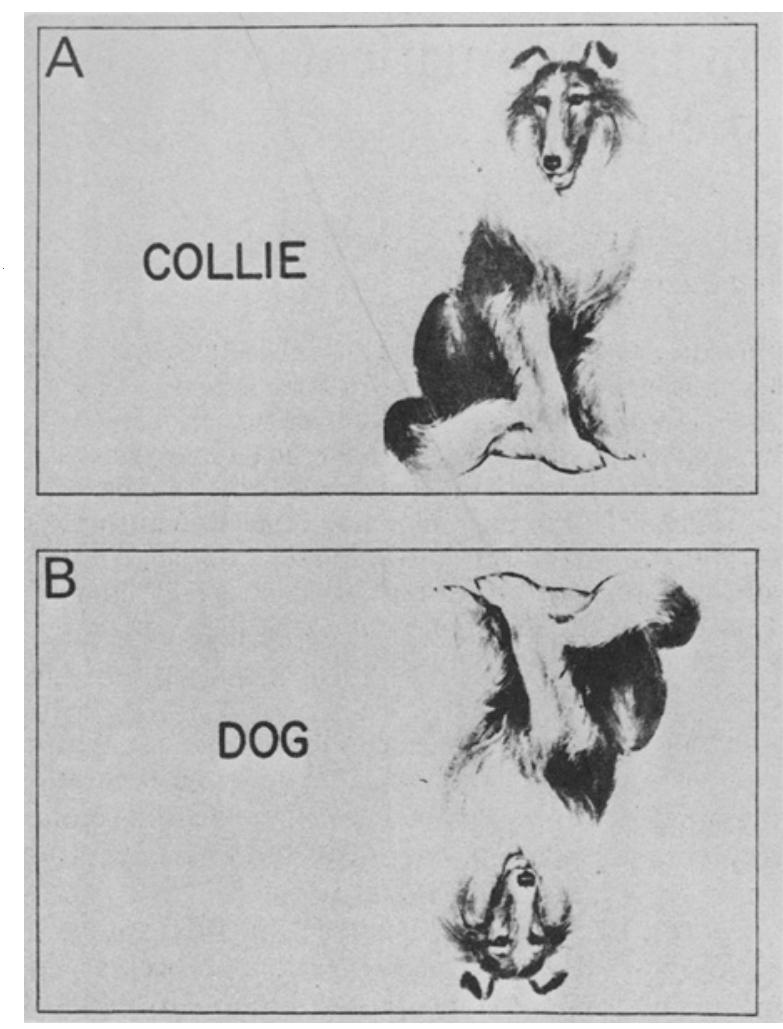

Flgure 1. (A) Example of word-picture match at specific level of categorization. (B) Example of word-plcture match (with picture rotated) at general level of categorization.

structural and analytic processes was obtained by Hock (1973), and subsequently replicated by Hock and Ross (1975). In both these studies, a significant correlation was obtained between the effects of symmetry (symmetical vs. asymmetrical patterns) and rotation (familiar vs. rotated-familiar patterns) on "same" reaction time. Based on the hypothesis that symmetry would serve as a rule for structuring the information in a stimulus into a well-organized whole, subjects with large symmetry effects (as well as large rotation effects) were inferred to emphasize a structural mode of processing. Subjects with small symmetry effects (as well as small rotation effects), who did not use symmetry as a structural rule, were inferred to emphasize an analytic mode of processing. Further evidence that analytic subjects attend to the featural parts of a stimulus, while structural subjects organize the parts of the stimulus into wholes, was obtained by Hock, Gordon, and Marcus (1974), who found that analytic subjects (small rotation effects) detect embedded figures more rapidly than structural subjects (large rotation effects).

In a subsequent study comparing reaction time of ' physical matches (e.g., AA) and name matches (e.g., aA), Hock, Gordon, and Gold (1975) obtained evidence that analytic subjects (small rotation effects) base their "same" responses to physically identical stimuli on a comparison of verbal codes, while structural subjects (large rotation effects) base their physical matches on a perceptual comparison. The propensity of analytic subjects to code visual information verbally was consistent with Bruner's (1957) contention that our perceptual knowledge is organized to achieve efficiency in stimulus identification. The present study examines Bruner's further contention that the efficiency is achieved by attending to the minimal number of features necessary to infer the categorical identity of a stimulus.

In the experiments reported in this paper, individual differences in stimulus identification were investigated in a task requiring subjects to indicate whether there was a match between a word and a simultaneously presented picture. The word paired with each picture was varied with respect to the specificity of categorization that was required. For example, when the pictured object was a collie, the matching word was either "collie" or "dog" (mismatches for this picture were the words "poodle" and "bird"). If it is assumed that the defining features for a superordinate category are those features that are common to (i.e., the mathematical intersection of) the defining features for each of its subordinate categories, then fewer features must be detected in order to place a stimulus in a superordinate category (e.g., dog) compared with its subordinate categories (e.g., collie, poodle).

On this basis, it was hypothesized that subjects emphasizing analytic processes would exhibit categorical inference in identifying the pictures by having faster word-picture matches for general than for specific category names. Since subjects emphasizing structural (synthetic) processes were expected to attend to the entire stimulus configuration, it was hypothesized that their word-picture matches would be unaffected by whether the word was a specific or a general category name. The overall hypothesis, therefore, was that the level of categorization indicated by the word would affect the time required for the word-picture matches of analytic but not for structural subjects.

\section{EXPERIMENT I}

\section{Method}

Stimuli. The stimuli used in this experiment were simultaneously presented word-picture pairings. The picture, which was always presented on the right side of the stimulus display, was normally oriented on half the trials and rotated $180^{\circ}$ into an unfamiliar orientation on the remaining trials. None of the pictures were invariant under a $180^{\circ}$ rotation. The pictures used in the experiment were of dogs (poodle, collie), birds (owl, eagle), automobiles (stationwagon, Volkswagen), boats (canoe, motorboat), hats (cowboy hat, baseball cap), shoes (sneaker, mocassin), chairs (high chair, rocking chair), lamp (lantern, desk lamp, buildings (barn, church), and trees (palm tree. pine tree). The 
accompanying word. which was never rotated. always remained on the picture's left. On half the trials, the word properly identified the picture: for the other half, the word did not match the picture. Half the words were specific category names (e.g.. collie) and half were more general category names (e.g.. dog). When the specitic names did not match the picture, the word (e.g., poodle) and the picture (e.g., collie) always belonged to the same superordinate category. When the general names did not match the picture (e.g., any kind of dog). the word was always another general category name (e.g. bird)..$^{1}$ Examples of word-picture stimuli are presented in Figure 1.

Design. A total of 160 stimuli were assigned to the orthogonal combination of three experimental variables: normal vs. rotated orientation (picture), specitic vs. general category name (word), and same vs. different (word-picture match), with 20 stimuli falling in each combination. The stimuli were presented in a randomly mixed sequence. Preceding the experimental trials, subjects were given 16 practice trials with stimuli that were not used for the experimental trials.

Procedure. The stimuli were back-projected onto a translucent screen with a Carousel projector, and the exposure duration was controlled by a tachistoscopic shutter. A visual angle of about 6.0 deg was subtended by the entire display, the picture and word each subtending a visual angle averaging about 2.6 deg. ${ }^{2}$ Each stimulus was presented for a duration of $3 \mathrm{sec}$. unless the subject responded, whereupon the display was terminated. The subjects were required to press a button whenever the word (either a specific nane or a general namel properly identified the pictured object. When there was no match betueen the word and picture, the subjects were not 10 respond in any way. The subjects were instructed to respond as quickly as possible, within the context of keeping their errors to a minimum. The reaction time from the onset of the stimulus display was the primary dependent measure.

Bufore beginning the reaction time trials, the 20 pictures used in the experiment were shown successively to the subjects, who were asked to identify them. If their response was a category name. they were supplied with the appropriate specitic name. If their response was a specific name that did not correspond with the specific name used in the experiment. the specitic name that would be used was provided.

Subjects. Twenty-four undergraduate students at Florida Atlantic University were paid $\$ 1.75$ for participating in an experimental session lasting about $30 \mathrm{~min}$.

\section{Results}

The mean reaction time and errors for Experiment $I$ are presented in Table 1 . The effect on "same" reaction time of rotating the pictures into an unfamiliar orientation was significant, $F(1,23)=$ $42.31\left(\mathrm{MS}_{\mathrm{e}}=996\right), \mathrm{p}<.005$. as was the difference in reaction time between specific and general category names. $F(1.23)=38.84\left(\mathrm{MS}_{\mathrm{e}}=1648\right), \mathrm{p}<.005$. The interaction between the effect of rotation and the type of word (specific vs. general) was insignificant.

Table 1

Experiment I: Mean Reaction Time (in Milliseconds) and Percentage Errors for Word (Specific vs. General Category Name) to Picture (Normal vs. Rotated Orientation) Matches

\begin{tabular}{|c|c|c|c|c|c|c|c|}
\hline & \multicolumn{3}{|c|}{ Reaction Time } & \multicolumn{4}{|c|}{ Errors } \\
\hline & \multirow[b]{2}{*}{$\mathbf{S}$} & \multirow[b]{2}{*}{ G } & \multirow[b]{2}{*}{$S-G$} & \multicolumn{2}{|c|}{ Commissions } & \multicolumn{2}{|c|}{ Omissions } \\
\hline & & & & S & G & $\mathrm{S}$ & $\mathrm{G}$ \\
\hline $\operatorname{Normal}(\mathrm{N})$ & 759 & 717 & 42 & 10.0 & .6 & .6 & 0 \\
\hline Rotated (R) & 810 & 750 & 60 & 6.3 & .2 & 0 & .6 \\
\hline $\mathbf{R}-\mathbf{N}$ & 51 & 33 & & & & & \\
\hline
\end{tabular}

Note $-\mathrm{S}=$ specific, $G=$ general

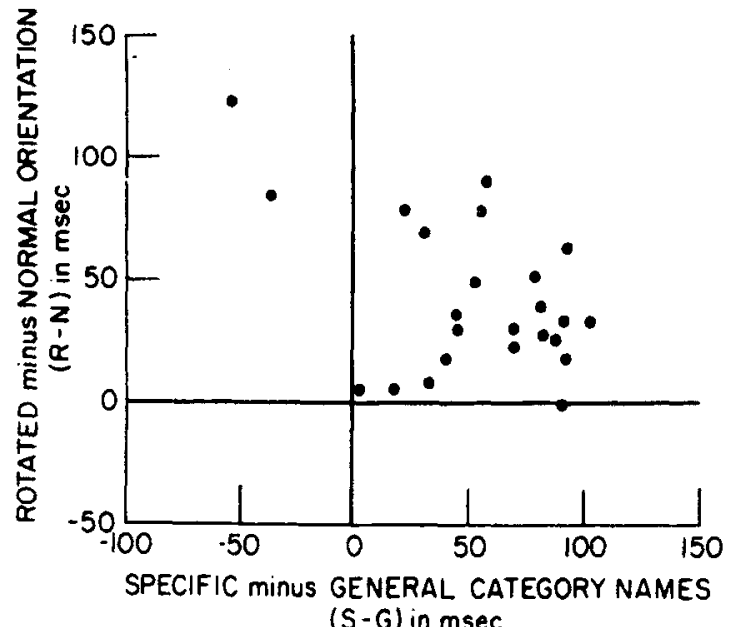

Flgure 2. Scattergram for word-picture "same" responses of Experiment I. Each dot represents one subjects.

$F(1,23)=2.12\left(\mathrm{MS}_{\mathrm{e}}=854\right), \mathrm{p}>.05$. The errors consisted almost entirely of errors of commission, almost all of which were obtained for the specific category names. The high error rate for the specific name matches suggests that the difference in reaction time between the specific and general matches would have been still larger had the subjects adopted a more conservative speed-accuracy criterion for the specific category names.

The hypothesis for this experiment was that subjects emphasizing analytic processes would have faster word-picture matches for general than for specitic category names, while word-picture matching time would be unaffected by the categorical level of the word for subjects emphasizing structural processes. Individual differences in processing were determined from the effect of rotating the pictures into an unfamiliar orientation: emphasis on analytic processes was inferred for subjects with small rotation effects, while emphasis on structural processes was inferred for subjects with large rotation effects.

The correlation between the effect of rotation on reaction time (R-N) and the difference in reaction time between specific and general category names (S-G) was significant, $r=-0.46, p<.05$ (see Figure 2 for scattergram). ${ }^{3}$ This correlation, however, was suppressed by differences in performance level between the subjects. For example, the subjects whose data fell close to the origin of Figure 2 tended to respond relatively rapidly in all the stimulus conditions, resulting in their having proportionally small rotation (L-N) and type-of-word (S-G) effects relative to slower subjects. When individual differences in performance level, as measured by each subject's average reaction time for the normally orientated pictures, was partialled out, the correlation coefficient increased to $\mathrm{r}=-0.52, \mathrm{p}<.02$.

In order to examine the results of this experiment 
Table 2

Experiment I: Mean Reaction Time (in Milliseconds) and Percentage Errors for Word-Picture Matches, With Subjects Divided According to Individual Differences in Processing

\begin{tabular}{|c|c|c|c|c|c|c|c|}
\hline & \multicolumn{3}{|c|}{ Reaction Time } & \multicolumn{4}{|c|}{ Errors } \\
\hline & & & & Comr & sions & Omi & ions \\
\hline & $S$ & $\mathbf{G}$ & $S-G$ & $\mathrm{~S}$ & $\mathrm{G}$ & $\mathrm{S}$ & G \\
\hline & \multicolumn{7}{|c|}{12 Analytic Subjects } \\
\hline $\begin{array}{l}\text { Normal (N) } \\
\text { Rotated (R) }\end{array}$ & $\begin{array}{l}749 \\
772\end{array}$ & $\begin{array}{l}694 \\
706\end{array}$ & $\begin{array}{l}55 \\
66\end{array}$ & $\begin{array}{l}9.6 \\
6.7\end{array}$ & $\begin{array}{l}1.3 \\
0\end{array}$ & $0^{.8}$ & $\begin{array}{l}0 \\
.4\end{array}$ \\
\hline \multirow[t]{2}{*}{$\mathbf{R}-\mathbf{N}$} & 23 & 12 & & & & & \\
\hline & \multicolumn{7}{|c|}{12 Structural Subjects } \\
\hline $\begin{array}{l}\text { Normal (N) } \\
\text { Rotated (R) }\end{array}$ & $\begin{array}{l}770 \\
848\end{array}$ & $\begin{array}{l}739 \\
794\end{array}$ & $\begin{array}{l}31 \\
54\end{array}$ & $\begin{array}{r}10.4 \\
5.8\end{array}$ & $\begin{array}{l}.4 \\
.4\end{array}$ & $0^{.4}$ & $\begin{array}{l}0 \\
.8\end{array}$ \\
\hline $\mathbf{R}-\mathbf{N}$ & 78 & 55 & & & & & \\
\hline
\end{tabular}

Note $-S=$ specific, $G=$ general

more closely, the subjects were divided into two groups (see Table 2). The 12 subjects with the largest rotation effects were placed in one group (structural), and the 12 subjects with the smallest rotation effects were placed in the second group (analytic). As in previous studies (Hock, 1973; Hock, Gordon, \& Gold, 1975: Hock, Gordon, \& Marcus, 1974), analytic subjects tended to be faster than structural subjects. However, the difference in reaction time between the two groups, for the normally oriented pictures, was not significant. $t(22)<1$. An examination of the errors indicated a similar distribution of omission and commission errors for the two groups of subjects. There was no indication that the individual differences obtained in this experiment were the result of differential speed-accuracy criteria for the two groups of subjects.

Although the obtained correlation was significant and in the hypothesized direction, it can be seen from Figure 2 that the scattergram was shifted to the right with respect to the predicted results. That is, although the level of categorization (specific vs. general) had a smaller effect on structural than on analytic subjects, the structural subjects were nonetheless faster on general than on specific matches [the difference was significant, $\mathrm{t}(11)=3.14, \mathrm{p}<.01]$. A possible explanation for this shift was the fact that the general category words comprised an average of 3.1 fewer letters than the specific category words. Thus, the time required for reading the words may have introduced a constant difference in reaction time between the specific and general category names.

Support for this possibility was obtained in an experiment that used the words of Experiment $I$ in a task requiring word-word comparisons. Half the trials required "same" responses for either specific words (e.g., COLLIE COLLIE) or general words (e.g., DOG DOG). The other half were "different" trials, for which no response was required (e.g., COLLIE POODLE; DOG BIRD). A total of 160 stimuli were presented in a randomly mixed sequence. The procedure was the same as in Experiment I. For a group of 12 subjects, the mean "same" reaction time was $737 \mathrm{msec}$ for the specific category names and $614 \mathrm{msec}$ for the general category names. The difference between the two types of word was significant, $t(11)=8.01, p<.001$. While this result supported the hypothesized word-length effect, there was no way to estimate the amount of extra time this contributed to the word-picture matches for the specific category names in Experiment I. A further experiment was required in order to control for the effect of word length.

\section{EXPERIMENT II}

This experiment was similar to Experiment 1, the main difference being that stimuli were selected such that the difference in word length between the specific and general category names was minimized $(0.4$ letters per word as compared with 3.1 in Experiment 1).

\section{Method}

Stimuli. The pictures used in this experiment were of dogs (poodle, collie), birds (owl. eagle). trees (palm, pine), buildings (barn, church), boats (canoe, motorboat), automobiles (stationwagon, Volkswagen), males (boy, man), and females (girl. woman). Pairings of words and pictures were as in Experiment $I$.

Design. The design of this experiment differed somewhat from that of Experiment 1. A total of 256 stimuli were assigned to the eight orthogonal combinations of normal vs. rotated orientation, specific vs. general category name, and same vs. different word-picture match. Each of these eight combinations was equally represented. in random order, within four blocks of 32 stimuli. The four blocks were then presented in one of four orders, according to a Latin square design, to four groups of subjects. For each group, the same sequence of blocks was presented twice, so that each subject worked on eight blocks of 32 trials. Preceding the above experimental trials were 32 practice trials corresponding to the last block of trials in the experimental sequence for that subject.

Procedure. The only difference from Experiment I regarding the procedure was that each stimulus was presented for a $1.0-\mathrm{sec}$ duration. Responses not occurring within this interval were counted as omission errors. The exposure was reduced from the 3.0-sec duration of Experiment $I$ in order to minimize the distortive effect of occasional long reaction times.

Subjects. Thirty-two unpaid undergraduate students at Florida Atlantic University voluntarily served as subjects in this experiment.

\section{Results}

Included in this experiment, but not the preceding one, were categories involving males (boy, man) and females (girl, woman). The implicit assumption in including these categories was that "boy" and "girl" were subordinate categories of "male" and "female." This assumption, however, was challenged by the insistence of many subjects (following the experimental session) that "girl" was a proper category name for females of any age. Their contention was consistent with the data: when the word "girl" was paired with a picture of a woman, the 
Table 3

Experiment II: Mean Reaction Time (in Milliseconds) and Percentage Errors for Word (Specific vs. General Category Name) to Picture (Normal vs. Rotated Orientation) Matches

\begin{tabular}{|c|c|c|c|c|c|c|c|}
\hline & \multicolumn{3}{|c|}{ Reaction Time } & \multicolumn{4}{|c|}{ Errors } \\
\hline & \multirow[b]{2}{*}{$S$} & \multirow[b]{2}{*}{$\mathrm{G}$} & \multirow[b]{2}{*}{$S-G$} & \multicolumn{2}{|c|}{ Commissions } & \multicolumn{2}{|c|}{ Omissions } \\
\hline & & & & $\mathrm{S}$ & $\mathrm{G}$ & $\mathrm{S}$ & G \\
\hline Normal (N) & 630 & 615 & 15 & 9.2 & .9 & 1.7 & 1.6 \\
\hline Rotated (R) & 669 & 645 & 24 & 7.4 & .8 & 4.9 & 1.2 \\
\hline$R-N$ & 39 & 30 & & & & & \\
\hline
\end{tabular}

Note $-S=$ specific,$G=$ general

rate of commission errors was $45.3 \%$, as compared with $7.8 \%$ for the remaining specific category names. This error rate was maintained despite the fact that the subjects received corrective feedback after each error. Further evidence of semantic difficulty with the female-male categories was indicated by the relatively high rate of omission errors for female and male general category names $(4.1 \%$ as opposed to $1.4 \%$ for the remaining general category names). Since this experiment was concerned with the effect of categorical information on visual processing, and not with anomalies in the semantic structure of male-female terms, the data for the male-female categories were excluded from the analyses that follow. 4

The mean reaction times and errors for Experiment II, excluding the male-female cattgories, are presented in Table 3 . The effect of rotating the pictures into an unfamiliar orientation, $F(1,31)=$ $150.46\left(M S_{\mathrm{e}}=258\right)$, and the difference in reaction time between specific and general category names, $\mathrm{F}(1,31)=15.81 \quad\left(\mathrm{MS}_{\mathrm{e}}=733\right)$, were significant. $p<.005$. The interaction between the effect of rotation and the type of word (specific vs. general), $\mathrm{F}(1,31)=1.84\left(\mathrm{MS}_{\mathrm{e}}=372\right), \mathrm{p}>.05$, was insignificant. As in Experiment $I$, errors were primarily errors of commission obtained when the words were specific category names.

The correlation between the effect of rotation on reaction time and the difference in reaction time between specific and general category names was significant, $\mathrm{r}=-0.47, \mathrm{p}<.05$, replicating the results of Experiment I (see Figure 3 for scattergram). When individual differences in performance level, as measured by each subject's average reaction time for the normally oriented pictures, was partialled out, the correlation coefficient was unchanged.

In order to examine the results of Experiment II more closely, the subjects were divided into two groups (see Table 4). The 16 subjects with the largest rotation effects were placed in one group (structural), and the 16 subjects with the smallest rotation effects were placed in the second group (analytic). As can be seen from Table 4, the hypotheses for the experiment were supported. For the subjects emphasizing analytic processes, the mean difference in reaction time between specific and general category names (S-G) was relatively large $(31 \mathrm{msec})$ and significant, $t(15)=$ 3.74. $p<.01$. For the subjects emphasizing structural processes, the difference between the specific and general category names was small $(8 \mathrm{msec})$, though it did fall only slightly short of significance, $t(15)=$ 2.12, at the .05 level.

As in Experiment $I$, there was no indication that the individual differences obtained in this experiment were the result of differential speed-accuracy criteria for the two groups of subjects. However, the high rate of commission errors obtained for the specific category names presented a potential problem in interpreting the reaction time data. That is, it is conceivable that the small difference in reaction time between specific and general category names $(S-G)$ for the structural subjects would not have been obtained had they adopted more conservative speed-accuracy criteria for the specific category names. This possibility, however, was not supported by the data. For the eight subjects in the structural group with less

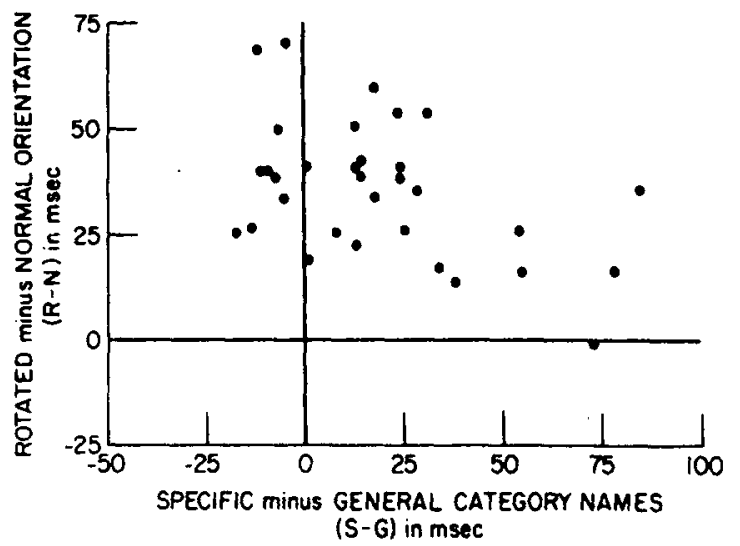

Figure 3. Scattergram for word-picture "same" responses of Experiment II. Each dot represents one subject.

Table 4

Experiment II: Mean Reaction Time (in Milliseconds) and Percentage Errors for Word-Picture Matches, With Subjects Divided According to Individual Differences in Processing

\begin{tabular}{|c|c|c|c|c|c|c|c|}
\hline & \multicolumn{3}{|c|}{ Reaction Time } & \multicolumn{4}{|c|}{ Errors } \\
\hline & \multirow[b]{2}{*}{$S$} & \multirow[b]{2}{*}{ G } & \multirow[b]{2}{*}{$S-G$} & \multicolumn{2}{|c|}{ Commissions } & \multicolumn{2}{|c|}{ Omissions } \\
\hline & & & & $S$ & $\mathbf{G}$ & $\mathbf{S}$ & $\mathrm{G}$ \\
\hline & \multicolumn{7}{|c|}{12 Analytic Subjects } \\
\hline $\operatorname{Normal}(\mathbf{N})$ & 644 & 613 & 31 & 9.6 & .8 & 2.3 & 1.0 \\
\hline Rotated (R) & 666 & 636 & 30 & 8.6 & 1.0 & 4.9 & 1.0 \\
\hline \multirow[t]{2}{*}{$R-N$} & 22 & 23 & & & & & \\
\hline & \multicolumn{7}{|c|}{12 Structural Subjects } \\
\hline Normal (N) & 615 & 617 & -2 & 8.9 & 1.0 & 1.0 & 2.1 \\
\hline Rotated (R) & 672 & 654 & 18 & 6.3 & .5 & 4.9 & 1.3 \\
\hline $\mathrm{R}-\mathrm{N}$ & 57 & 37 & & & & & \\
\hline
\end{tabular}

Note $-S=$ specific, $G=$ general 
than three commission errors for the specific category names (their average error rate was $2.3 \%$ ), the mean difference in reaction time between the specific and general category names (S-G) was only $1 \mathrm{msec}$. That is, the subjects with low error rates (conservative speed-accuracy criteria) for the specific category names more closely conformed to the hypothesized result than the subjects with higher error rates.

Unlike Experiment $I$, and most previous studies, the analytic subjects were not faster than the structural subjects on the normally oriented pictures. This inconsistency may be due to task differences compared with previous studies; the pictures used here were generally more complex than the stimuli used previously, and most of the earlier studies involved matching physically identical stimuli rather than words and pictures.

Finally, it could be argued that the difference in word-picture matching time between specific and general category names was biased by differences in frequency of usage. That is, the verbal codes for the pictures and/or words may have been relatively more "available" for the more frequent general category names than the less frequent specific category names. In order to examine this possibility, four lists of 96 words were formed, two lists composed of the general category names and two lists composed of the specific category names (the words for the male-female categories were excluded from all the lists). Twelve subjects were instructed to read each list (including an additional practice list) as quickly as possible, and the time required to read the entire list was recorded. The difference in reading time between the specific and general category names was $12 \mathrm{msec} /$ word, which was insignificant. $t(11)=1.31, \mathrm{p}>.05$. This result, which involved the effect of frequency of usage on the verbal coding of the words, together with Wingfield's (1968) finding that frequency of usage did not affect either picture-picture matches or word-picture matches (the word preceded the picture) suggests that the results of this experiment were not biased by frequency of usage effects.

\section{DISCUSSION}

The results of Experiments I and II supported the hypothesized individual differences in the effect of categorical information on the identification of familiar visual stimuli. In Experiment II, which controlled for differences in word-length between specific and general category names, it was found that subjects emphasizing analytic processes had faster word-picture matches for general than for specific category names. This finding, which supported the hypothesis that analytic subjects attend to only the minimal number of features necessary to infer the categorical identity of the pictures, had two further implications. First, it suggested that feature processing is self-terminating for analytic subjects. If "same" responses had been based on an exhaustive feature analysis, the difference in reaction time between specific and general matches would not have been obtained. The results for analytic subjects also suggested that they selectively attend to orientationally invariant features and ignore orientationally dependent features in categorizing the pictures. Such orientationally invariant features could be global (e.g., symmetry, color) or a particular part of the picture (e.g., a subgrouping of elements forming a right angle). Of interest in this regard would be whether the specificity of a category is related to the type of feature that defines the category. Thus, orientationally invariant global features may define relatively general categories, orientationally invariant parts may define more specific categories, and orientationally dependent features may define very specific categories (a particular object or person). This sort of parallel would be consistent with Bruner's (1957) contention that categorization proceeds in stages from a relatively "open" feature search to a "closed" search for specific features to confirm a preliminary categorization.

For subjects emphasizing structural processes, there was little difference in reaction time between specific and general category names. This supported the hypothesis that structural subjects attend to all the information in the pictures, regardless of the level of categorization specified by the accompanying word. While the results for analytic subjects suggested that their processing of features was self-terminated, the results for structural subjects suggested exhaustive processing. Exhaustivity, in this case, would imply that all the detailed parts of the stimulus are organized into a whole and not that the stimulus is analyzed into a complete set of features.

The results obtained for analytic subjects converged with evidence obtained in tasks requiring subjects to search for a target among an array of numbers or letters. As Neisser (1963) has pointed out, if subjects search quickly enough they can correctly reject nontargets with little phenomenal experience of the letters or numbers they are rejecting. This indication that subjects attend to a minimal amount of stimulus information during high-speed search suggests that they are processing information analytically, inferring that a stimulus is a nontarget by testing a relatively small number of distinctive features. In terms of the present study, this possibility would be supported if search rates were faster for general than for specific levels of categorization. Just this result, in fact, has been obtained by Ingling (1972), who found that subjects rejected nontargets more rapidly when they were looking for a letter against a background of numbers (the nontargets could be categorized at a general level as numbers) than when they were looking for a letter against a background of other letters (the 
nontargets had to be categorized at a specific level as particular letters).

Although the results of the present research were consistent with the hypothesis that analytic subjects tend to minimize the amount of stimulus information to which they attend, it does not follow that they must invariably be faster than structural subjects. In the word-picture task used in this study, the analytic subjects may "save" processing time by attending to only the distinctive features in the pictures, but the time required to retrieve the categorical distinctive feature information associated with the word could more than compensate for the savings due to selective attention. For tasks requiring "same" responses to physically identical stimuli, the savings in processing time resulting from selective attention to distinctive features could be compensated for by the time required for the analytic subjects to generate a verbal code for the stimuli (Hock, Gordon, \& Gold, 1975). At this point, there is no way to satisfactorily separate out these components of processing time, and therefore no way to predict whether analytic subjects will be faster than structural subjects.

To summarize, the individual differences methodology used in this study has provided evidence for alternative modes of identifying familiar visual stimuli, and therefore given simultaneous support to two apparently conflicting theories (Bruner, 1957; Neisser, 1967). The usefulness of this methodology goes beyond the existence of individual differences, which are important in and of themselves. More significantly, the methodology suggests that many conflicts in cognitive theory may be resolvable, not by finding evidence that favors one theory at the expense of the other, but by showing within the confines of a neutral task that one theory applies to the mode of processing adopted by some subjects, while the other theory applies to a different mode of processing adopted by other subjects.

\section{REFERENCES}

Bruner, J. S. On perceptual readiness. Psychological Review, 1957, 64, 123-152.

Hock, H. S. The effects of stimulus structure and familiarity on same-different comparison. Perception \& Psychophysics, $1973,14,413-420$.

Hock, H. S., Gordon, G. P., \& Gold, L. Individual differences in the verbal coding of familiar visual stimuli. Memory \& Cognition, 1975, 3, 257-262.

Hock. H. S., Gordon, G. P., \& Marcus, N. Individual differences in the detection of embedded figures. Perception \& Psychophysics, 1974, 15, 47-52.

Hock, H. S., \& Ross, K. The effect of familiarity on rotational transformation. Perception \& Psychophysics, 1975, 18, 15-20.

INGLING, N. W. Categorization: A mechanism for rapid information processing. Journal of Experimental Psychology, 1972, 94, 239-243.

NeIsSER, U. Cognitive psychology. New York: Appleton-CenturyCrofts, 1967.

WINGFIELD. A. Effects of frequency on identification and naming of objects. American Joumal of Psychology, 1968, 81, 226-234.

\section{NOTES}

1. For the word-picture mismatches at general levels of categorization, the word "bird" was paired with the pictures of dogs and the word "dog" was paired with pictures of birds. General level mismatch pairings for the remaining pictures were: automobileboat. hat-shoe, chair-lamp, and building-tree.

2. In order to minimize the visual angle of the words, some of the specitic category names involving two words (e.g., rocking chair) were presented one word above the other.

3. Although the effect of rotating the picture $(R-N)$ and effect of the category level of the word $(S-G)$ include common components (e.g., specific word paired with normally oriented picture), (R-N) and (S-G) are logically orthogonal. In this way, the correlational analysis is based on the same assumptions of statistical independence that are ordinarily applied to analysis of variance.

4. Of further interest were subject-sex differences with regard to the male-female category names. The rate of commission errors when the word "girl" was paired with a picture of a woman was $26.8 \%$ for female subjects and $59.7 \%$ for male subjects. These sex differences with regard to the semantics of sex terms will be the subject of further investigation.

(Received for publication July 8, 1975; revision accepted September 18, 1975.) 